\title{
XLI. On fulminating gold
}

\section{J.B. Van Mons}

To cite this article: J.B. Van Mons (1816) XLI. On fulminating gold, Philosophical Magazine Series 1, 47:215, 176-179, DOI: 10.1080/14786441608638829

To link to this article: http://dx.doi.org/10.1080/14786441608638829

曲 Published online: 27 Jul 2009.

Submit your article to this journal $2 \pi$

Џ Article views: 2

Q View related articles $₫$ 
pretends to argue, I feel myself completely justified in relinquishing the present controversy: it wonld indeed he litigious in the extreme for me to continue to oppose one whe writes not against me, but against the new system (that metalic salts are super-salts with excess of oxide) of which your correspontent G. S. is the author.

Yours obediently,

Lontion, March 6, 1216.

H.

\section{On Fulninating Gold. By J. B. FAx Moss, of Brussels*.}

\section{T} HERr is a preparation of gold which presents much interest, and on which the nephew of the illustrious Driessen has published a very good essay :-I mean fulminating gold. This compound may be procured by all the methods of putting ammonia in reaction on dissolved gold; by aqua regia, with which we precipitate gold by the help of a soluble oside, and by the muriate of gold which we decompose by means of ammonia; by the oxide, of gold which we treat with ammonia, either gaseous or liquid or with any ammoniacal salt; and finally, by this same oxide, which we must kecp some time in a dark and damp place where the air is stagnant. I prepare it with most advantage by precipitating by potash a diluted solution of muriate of gold and muriate of ammonia. The precipitate is at first muriate of ammonia and gold; but the instant the ammonia becomes free, it is aurate of ammonia, called ammonium of gold, or fulminating gold, which is produced.

Fulminating gold is not decomposed by any acid, and it is not by the alkalies: nevertheless the first of these bodies disunite it by the adjunction of a hyperoxygenated muriate and liquid chlorine; without this adjunction, but with the assistance of heat, double muriate being produced. This happens from the combustibles having more energy than its metal. This compound is a salt in which the oxide of gold performs the finctions of acit, and the ammonia the function of an oxide: it is therefore, $I$ have already said, an aurate of ammonia, which exists by ax engagement the nore intimate as the oxygen of the gold, with vinich the oxygen of the ammonia is proportioned, presents more caloric to disjlace. The acids, in order to decompose this salt, ought either to take the ammonia from it or dissolve at once its two elemenis; but for the first effect it is necessary that the oxide of gold should be insulated from the oxygen wanting caloric, and tor the second effect it would be necessary

* Communicated by the Author. 


\section{On Fulminating Gold.}

that the acids should be able to dissolve oxide of this same quality, both which are absolutely impossibilities: and if such effects counc obtained, they would nultiply al infinitum the number of oxives, salts, and all other bodies having oxygen in combination.

Nevertheless fre and time, by adiling caloric to the oxygen of the gold, determine the disenragement of the principies of the fulminating goll and their being taken in solution : energetic conbustibles take from the yold the ssyen, with its letect of caloric, reduce its metal, and set ine anivunia at liberty : which explains low, in a glote of fure strongly hented, the fuininating gold is deomposed without fuiminating; which certainly would not hapien if the ball were platina, gold, or silver.

Ammonia has too little affinity with water to enable that liquid aided by heat to take it from the gold: nevertheless the heat in this case brings together the active principles of the compound; and this happens when we wash it with very hot water: the caloric displaces in the first place the ammonia from the oxide, the hydrogen from the azote, and the oxygen from the gold, and pressure disunites them by forming water. Fulminating gold does not detonate under water, for want of power to take the temperature requisite for this effect; and at a heat a little higher it resolves into its nearest principles, with the single exception of the case in which it has been washed too hot.

Carbon organized atd hydrogenated in the oils with ether and alcohol, as well as azote hydrogenated in ammonia, take up the oxide of gold from its solvents; which proves that this body acts more willingly by capacity than by intensity; and the oils may eren decompose the fulminating gold by taking up on one hand its oxide and on the other hand its ammonia. The oxide of gold which is separated from its solutions by the contact of the air-will it be aurate of organized azote, or oxide rendered insoluble by hyperoxidation? "In this last case, as these solutions are always with an excess of acid, the heating in those of the muriatic acid must determine the resumption in solution of the oxide under the formation of oxygenated muriatic acid.

The sulphuric acid highly concentrated decomposes fulminating gold by provoking the union between the principles of the water in order to appropriate to itself this water. The detonating compounds, which the same acid decomposes with an explosion, set the oxygen at liberty.

The fulminating gold is formed spontaneously in the same circumstances and by the same causes as the native nitrate of lime : it is the organized azote of the air hydrogenating itself into an oxidule which produces the first body, and the same

Vol. 47. No.215. March 1816. 
azote sub-hydrogenating itself into an acid which produces the second; and it may be presumed that the two actions take place simultaneously, or depend upon each other.

Gold in very minute division is dissolved in chlorine by the help of heat, and also when nitro-muriate of gold is partly decomposed by heat, then treated with muriatic acid, and evaporated to dryness. It is a brown substance, very deliquescent, which easily decomposes the water of the atmosphere, forming muriate of gold. I have not as yet examined it with precision.

\section{On Silver. [By the same.]}

It will not easily be believed, as Brugnatelli observes, that silver, which is a metal reducible per se, may be oxidized in the air, and at a heat which its oxide scarcely requires in order to be reduced. There must, therefore, be some other cause than oxidation which renders silver vitrifiable : and this cause will deserve examination. A very intense heat, or the electric fluid,-do they organize a portion of the oxygen of the primitive matter which forms the basis of the silver, in such a way as to originate an oxide different from the ordinary oxide of that metal ? This is a difficult question. Such an effect, by displacing hydrogen with the oxygen of the primitive matter, will compose a metal more hydrogenated, and which will be irreducible in the fire; and the oxidation will take place not by the oxygen of the air, but by that which is proper to the primitive matter.

We must inquire if the oxide of silver obtained by fire possesses other qualities than the oxide of the same metal obtained by the acids; and in the contrary case, we must ascertain if the reagents employed in this examination do not separate the supercombined hydrogen, proportioning at the same time the oxygen of the caloric to the degree of the new hydrogenation; or if, by oxidating the hydrogen of the oxide at the ordinary degree, they do not separate some of it from the water-two circumstances which will bring silver back to its first constitution.

We know already that the metals are oxidated by the electric Auid, as well in vacuo as in contact with the air. The experiments relative to the oxidation of silver by caloric alone Inust be undertaken on a very large scale in close vessels, on account of the volatility of the metal, and in vacuo: we mast particularly ascertain if the metal, in returning to its primitive state, undergoes a loss of weight.

If the fact of the mineral organization of the primitive matter of a metal by the electric fluid, or by a red heat, is confirmed; we may explain how in aërolites the metals may be artificially composed, 
composed, and the nature as well as the mode of organization of the primitive matter of the globe will thereby be elucidated; and we shall extract oxygen from a substatice in which its presence is least expected. This fact will, besiles, denote that the metals the oxides of which are reducible by fire, owe this cuality to at smaller dose of oxygen, which is at the same time the cause of their greater weight.

Juncker had already ascertained that at a very intense heat silver is converted into a fused oxide ; and Richter mentions the fact of a considerable quantity of silver which a modern alchemist had kept in the fire several years, being thereby transformed into a mass of fused oxide. M. Richter having obtained this mass, trier to reduce it, first by heat and afterwards by the usual reducers, but he was able to extract only a very small portion of metal.

Silver, on being organized, may also become an acidifiable combustible; for between an oxide and such a body the distance is not great : and if azote is hydrogenated into ainmonin, and if tellurium and arsenic, by an inverse action, from metals become acidifiable combustibles, silver may very well, on receiving câJeric, without losing hydrogen, become a double super-combination of this principle, and constitute either an acidifiable combustible or the oxide of a different metal, more energetic, because it has more hydrogen, and because with precautions we may obtain it reduced. This will be the second artificial metal, and others will soon follow, which, like ammonia, will be decomposable, because it will exist by composition, and in which caloric, favoured by a disposing affinity, may be substituted for the hydrogen of the new metallization, and allow the oxide of silver to be reduced, per se, into its metal : but hydrogen at a lesser heat might also be substituted for the water of the new metal, and thus present it to us reduced. How can we see oxide of silver resist reduction in the fire, without making such a phænomenon the subject of the most serious meditation?

XLII. On the Cosmogony of Moses, in Answer to Dr. Pritehard. By F. E-s.

To Mr. Tilloch.

SrR, - I Feel no inclination to involve Doctor Prichard in controversy; but as he has had the advantage of fully stating the grounds of his opinions, given in your Magazine, relative to the Mosaic cosnogony, it will, I presume, be allowed me briefly to examine whether he has succeeded in removing the objections againat them indicated in my former letter. 